\title{
Heterogeneous Expectations, Optimal Monetary Policy, and the Merit of Policy Inertia
}

\author{
Emanuel Gasteiger ${ }^{\mathrm{a}, *}$ \\ ${ }^{a}$ Instituto Universitário de Lisboa (ISCTE - IUL), BRU - IUL, Department of Economics, \\ Av. ${ }^{a}$ das Forças Armadas, 1649-026 Lisboa, Portugal
}

\begin{abstract}
The design and analysis of optimal monetary policy is usually guided by the paradigm of homogeneous rational expectations. Instead, we examine the dynamic consequences of design and implementation strategies, when the actual economy features expectational heterogeneity. Agents have either rational or adaptive expectations. Consequently the central bank's ability to achieve pricestability under heterogeneous expectations depends on its objective and implementation strategy. An expectations-based reaction function, which appropriately conditions on private sector expectations, performs exceptionally well. However, once the objective introduces policy inertia, popular strategies can fail. These results call for new implementation strategies under interest rate stabilization.
\end{abstract}

JEL Classification: E52, D84

Keywords: Optimal Monetary Policy, Heterogeneous Expectations, Policy Inertia

\footnotetext{
${ }^{4}$ I gratefully acknowledge the extensive and constructive comments and suggestions of two anonymous referees and the editor that I incorporated into the substantially revised paper. I am also indebted to Gerhard Sorger and Robert Kunst for sound supervision and patience. Moreover, I am grateful to Bennett T. McCallum for the insightful correspondence about calculating solutions to rational expectations models as well as to Seppo Honkapohja, Torsten Lisson, Karl Schlag, Shoujian Zhang, and the participants of the Graduate Seminar at the University of Vienna, the seminar at City University London, and the 6th PhD Presentation Meeting of the Royal Economic Society for many helpful comments. Any remaining errors are the responsibility of the author. Financial support from Fundação para a Ciência e a Tecnologia (PEst-OE/EGE/UI0315/2011) is acknowledged.

${ }^{*}$ Corresponding author: Av. ${ }^{a}$ das Forças Armadas, 1649-026 Lisboa, Portugal, +351-926785229, emanuel.gasteiger@iscte.pt

Email address: emanuel.gasteiger@iscte.pt (Emanuel Gasteiger)
} 


\section{Motivation}

A considerable amount of literature is concerned with the design and implementation of optimal monetary policy within the New Keynesian (NK) framework. Prominent examples are the analyses of Clarida, Galí, and Gertler (1999), Woodford (2003), and Galí (2008). A core assumption in these studies is that homogeneous agents adopt the rational expectations hypothesis (REH). In these works the central bank usually faces a linear-quadratic policy problem, where the quadratic objective corresponds to a second-order approximation of household utility. Once the optimal policy is specified, an implementation strategy is chosen. Analysts then ask, whether a specific implementation strategy can yield local determinacy, i.e., bring about a unique stationary rational expectations equilibrium (REE). ${ }^{1}$

Local determinacy, though not entirely uncontroversial, is associated with price-stability in current mainstream monetary economics. The issue of stable inflation itself is one of utmost importance, as the common transversality conditions in macroeconomic models rule out only explosions of real variables and not of nominal variables. Cochrane (2011) reemphasizes this point and questions determinacy as adequate means to ensure price-stability. ${ }^{2}$

Implementation strategies are frequently formulated as rules or reaction functions, in which the central bank's nominal interest rate is a linear function in inflation, output, and sometimes the lagged value of the nominal interest rate. Clearly, such reaction functions allow the policymaker to apparently relate its mandate to its policy instrument, which increases transparency and can make policy more coherent, credible, accountable, and easier to communicate.

\footnotetext{
${ }^{1}$ Determinacy most importantly rules out undesirable evolutions of endogenous variables such as large fluctuations. See for example Woodford (1999, p.69).

${ }^{2}$ For further discussion see McCallum (2009b,c) and Cochrane (2009). 
It is somewhat surprising that most considerations of optimal policy design and implementation are guided by the paradigm of homogeneous rational expectations (RE). An approach that uses a less restrictive assumption about expectations of homogeneous individuals is the learning theory. In this strand of literature it is assumed that individuals form their expectations adaptively and that they behave like econometricians when they forecast the future of prices and other variables. ${ }^{3}$ In studies such as Evans and Honkapohja (2003, 2006, 2010) or Duffy and Xiao (2007), the concept of learning serves as a robustness-check. Authors ask whether a unique stationary equilibrium, which the central bank is able to implement via optimal policy under homogeneous RE, remains stable if homogeneous agents have bounded rationality and learn. If so, the equilibrium is called expectationally stable and learning agents can coordinate on it.

From our perspective, this might be a comparison of two extreme and homogeneous types of expectation formation, but intermediate cases are also of interest. For empirical evidence that justifies the consideration of heterogeneity in expectations see, for instance, Branch (2004). Assenza, Heemeijer, Hommes, and Massaro (2013) also provide experimental evidence that suggests heterogeneity in expectations. Moreover, they find that a heterogeneous expectations switching model supports their evidence at the individual and aggregate levels.

As a consequence, one may ask whether agents' expectational heterogeneity is a challenge for optimal monetary policy design and related implementation strategies. We take up exactly this question and examine the conditions under which different objectives and the related implementation strategies allow a central bank to achieve determinacy. ${ }^{4}$ We consider this our primary contribution.

\footnotetext{
${ }^{3}$ See Evans and Honkapohja (2001) for a rigorous discussion of the learning theory.

${ }^{4}$ Note that Branch and Evans (2011) study simple and optimal discretionary monetary pol-
} 
In particular, the central bank minimizes a given quadratic loss function that punishes inflation and output gap deviations. We call this loss function the conventional objective. We then let the central bank solve its optimal monetary policy problem under commitment in an economy that features heterogeneous expectations à la Branch and McGough (2009). ${ }^{5}$ Subsequently, we examine the implementation of this policy by a so-called expectations-based reaction function. Our analysis can therefore be regarded as a further robustness-check for a widely discussed approach to optimal monetary policy design and implementation. We find that the expectations-based reaction function guarantees determinacy throughout the considered parameter space, as the central bank always responds stronger to the type of forecasts, which are more relevant to inflation pressures. This is a worthwhile result as heterogeneous expectations turn out to be an important source of economic instability under simple interest rate rules (see for example Branch and McGough 2009, Massaro 2013).

Subsequently, we repeat this analysis under misspecification of the true economic model in the design of optimal policy. In particular, we maintain the prevailing assumption that the central bank bases policy design on the REH paradigm. This approach is of practical relevance, as it may have important consequences for economic modelling. It is desirable to find an implementation for the optimal policy stance that renders the actual economy with heterogeneous expectations determinate, while the central bank is designing the optimal policy under the paradigm of the REH. In such a case, central bankers may be able to elaborate other aspects of policy analysis in a homogeneous RE version of the

icy in a heterogeneous expectations model with the focus on how equilibria with heterogeneous beliefs can arise.

${ }^{5}$ We do not consider the case of discretionary policy herein as we find the case of commitment more interesting due to the arguments about time-inconsistency going back to Kydland and Prescott (1977). 
model while guaranteeing price stability. Thus, they may be able to simply ignore possible structural and parameter uncertainty about the nature of private sector expectations in the process of design. The obvious advantage of the homogeneous $\mathrm{RE}$ version is that it is usually much easier to handle and to analyze. ${ }^{6}$

Our second key contribution is the finding that even an expectations-based reaction function based on the conventional objective is no longer a guarantee for determinacy in general, when the actual economy exhibits heterogeneous expectations. It is fair to say that the danger of triggering explosive paths of the price level is quite high, once non-rational agents become extrapolators. This result stands in contrast to the findings for homogeneous expectations and our main results. Therefore, we put a warning sign on this misspecification approach. Moreover, we also show that this policy cannot be time-consistent, at least in a deterministic version of the model. This strengthens our case for optimal monetary policy design based on a heterogeneous expectations model.

In the subsequent analysis, we augment the central bank's quadratic loss function by a term that makes interest rate stabilization (i.e., policy inertia) a desirable target for the central bank. We call this loss function the alternative objective and it introduces policy inertia into the implementation strategies. The consideration of this objective is based on the finding of Bullard and Mitra (2007), that policy inertia in simple rules can considerably improve the determinacy properties of the standard NK model.

Similar to the case before, the central bank solves its problem and is considered to implement its optimal policy either via an implicit instrument rule or via an

\footnotetext{
${ }^{6}$ In practice, central banks use so-called medium-scale DSGE models under the REH (see for example Smets and Wouters 2003, 2007) although they track private sector expectations and are aware of expectational heterogeneity. Christiano, Trabandt, and Walentin (2010) review the recent developments regarding the use of such models.
} 
expectations-based reaction function into the actual economy with heterogeneous expectations.

Our third key contribution is the finding that in the presence of expectational heterogeneity, policy inertia yields a poorer performance with regard to rendering the economy determinate. This questions the merit of policy inertia in the context of optimal monetary policy.

The remainder of the paper is organized as follows. In Section 2 we briefly describe the economic model and the notion of optimal policy employed in our study. Section 3 addresses the conventional objective. We also explain how we numerically analyze the dynamic properties of implementation strategies, make some comments on our calibration and discuss our main results. Section 4 discusses robustness to misspecification in policy design. Section 5 treats the alternative objective. Finally, Section 6 concludes and points out directions for further research.

\section{The Set-Up of the Analysis}

\subsection{The Economic Environment}

We assume a heterogeneous expectations reduced form NK economy as derived by Branch and McGough (2009). Aggregate demand and supply evolve according to

$$
\begin{aligned}
& x_{t}=\widehat{E}_{t} x_{t+1}-\sigma^{-1}\left(i_{t}-\widehat{E}_{t} \pi_{t+1}\right), \quad \text { and } \\
& \pi_{t}=\beta \widehat{E}_{t} \pi_{t+1}+\lambda x_{t} .
\end{aligned}
$$

The variable $x_{t}$ denotes period $t$ aggregate output gap, $i_{t}$ is the nominal interest rate controlled by the central bank, and $\pi_{t}$ is the rate of inflation. All variables 
are expressed as deviations from their mean. Note that we deliberately do not consider stochastic shocks, which arguably rules out important questions related to how the economy responds to aggregate exogenous shocks. ${ }^{7}$ The parameter $\sigma$ denotes the coefficient of relative risk aversion, which in this setting equals the inverse of the inter-temporal elasticity of substitution of private consumption. The parameter $\beta$ is the common discount factor and $\lambda$ is a combination of structural parameters that governs the sensitivity of inflation to changes in the output gap, see for example Clarida, Galí, and Gertler (1999, p.1667). $\widehat{E}_{t} z_{t+1}$ is the heterogeneous expectation for any aggregate variable $z_{t+1}$ as specified in Branch and McGough (2009, p.1038). ${ }^{8}$ Following the latter, the heterogeneous expectations operator has the particular property that $\widehat{E}_{t} z_{t+1}=\alpha E_{t}^{1} z_{t+1}+(1-\alpha) E_{t}^{2} z_{t+1}$.

Here $\alpha \in[0,1]$ is the share of agents that utilize the RE operator $E_{t}^{1} z_{t+1}=$ $E_{t} z_{t+1}$. The fraction $(1-\alpha)$ is not fully rational in the sense that these agents form expectations by using the forecasting model $E_{t}^{2} z_{t+1}=\theta E_{t}^{2} z_{t}=\theta^{2} z_{t-1}$, where the parameter $\theta$ governs the nature of the forecast, which can be purely adaptive $(\theta<1)$, naïve $(\theta=1)$, or extrapolative $(\theta>1)$. As a consequence, aggregate expectations for endogenous variables are given by

$$
\begin{aligned}
& \widehat{E}_{t} x_{t+1}=\alpha E_{t} x_{t+1}+(1-\alpha) \theta^{2} x_{t-1}, \quad \text { and } \\
& \widehat{E}_{t} \pi_{t+1}=\alpha E_{t} \pi_{t+1}+(1-\alpha) \theta^{2} \pi_{t-1} .
\end{aligned}
$$

\footnotetext{
${ }^{7}$ Branch and McGough (2009) derive (1) and (2) in a Yeoman-farmer economy. In case of a decentralized market-economy, it is far from clear how aggregate shocks affect individual income of ex-ante heterogeneous agents and whether (1) and (2) still remain valid. However, in a related study, we derive the very same structural equations including exogenous shocks in a decentralized market-economy.

${ }^{8}$ Note that Branch and McGough (2009) make use of an "axiomatic approach" and impose some assumptions that may appear restrictive to other scholars, but are a necessity to achieve the aggregate equations (1) and (2). Briefly, the assumptions that may be regarded as critical are the specification of higher order beliefs and the assumption that wealth dynamics do not matter for the evolution of aggregate variables. For a detailed discussion we refer the reader to Branch and McGough (2009).
} 
In what follows, we will close the model in each case with a different implementation strategy for monetary policy that is considered to be optimal.

From (3) and (4) it should become clear that past values of aggregate endogenous variables can affect aggregate demand and supply when RE and adaptive expectations coexist. This feature makes the model self-referential, i.e., expectations affect equilibrium outcomes and equilibrium outcomes affect expectations

and so on. As a result, implementation strategies that perform well in pure RE models may not necessarily do so under heterogeneous expectations.

\subsection{The Notion of Optimal Policy}

The purpose of this study is to discuss several strategies that seek to implement the optimal REE. By optimal we mean that the central bank is committed to the economic well-being of the individuals that populate the economy. Thus, the central bank should maximize the utility of individuals. Therefore, optimal policies assessed throughout the paper are based on the assumption that the central bank tries to minimize welfare losses caused by sizeable volatility in variables that matter for the utility of individuals.

In the adaptive learning literature it is common to assume that the central bank designs its policy under RE and subsequently implements the policy in the actual economy (see for instance Evans and Honkapohja 2006, 2010, Duffy and Xiao 2007). In contrast, optimal policy herein must be based on the correct model given by (1)-(2), and consequently also (3)-(4). 
The central bank therefore aims to minimize an ad hoc loss function ${ }^{9}$

$$
E_{t}\left\{\sum_{s=0}^{\infty} \beta^{s} \frac{1}{2}\left(\pi_{t+s}^{2}+\omega_{x} x_{t+s}^{2}+\omega_{i} i_{t+s}^{2}\right)\right\}
$$

subject to (1)-(2), where for the whole of this study, we assume that the central bank employs a commitment from a timeless perspective. ${ }^{10}$ Coefficients $\omega_{x}$ and $\omega_{i}$ are the relative weights that the central bank may attach to output and interest rate stabilization. Intuitively, the central bank is concerned about stabilizing inflation, the output gap, and its instrument over the entire planning horizon.

In Section 3 and 4 , we consider the case of $\omega_{i}=0$, which yields the conventional objective. Based on this, we discuss an optimal policy implementation strategy as analyzed in Evans and Honkapohja (2006, p.18), namely the expectations-based reaction function. Later on, in Section 5 we consider the case of $\omega_{i}>0$, which we call the alternative objective on the grounds that it is studied less frequently. This allows us to discuss the implementation strategy termed implicit instrument rule as discussed in Duffy and Xiao (2007). ${ }^{11}$ In Section 5 we also consider what we refer to as an alternative expectations-based reaction function. Evans and Honkapohja (2009b, p.158ff.) discuss it for discretionary optimal policy and homogeneous expectations.

The case of $\omega_{i}>0$ is of particular interest. Studies of simple monetary policy

\footnotetext{
${ }^{9}$ This ad hoc loss function serves as a proxy for a welfare loss function. For the case of $\alpha=1$, the latter can be derived by a second-order approximation of the utility function of the individuals, see Galí (2008, p.95ff.) or Woodford (2003, p.383ff.) for the case of $\omega_{i}=0$ and Woodford (2003, p.419ff.) for the case of $\omega_{i}>0$. Notice that as in Evans and Honkapohja (2006, p.18), we consider the case where the output gap target is zero to omit the problem of inflation bias.

${ }^{10}$ This requires that the central bank respects "the optimality conditions from the full intertemporal optimization under commitment, except for the current decision-making period" $s=$ 0, see Evans and Honkapohja (2006, p.16).

${ }^{11}$ For a pure RE version of the NK model, Woodford (2003, p.582ff.) and Giannoni and Woodford (2005, p.106ff.), present related analyses.
} 
rules such as Bullard and Mitra (2007) find that policy inertia in simple rules may be a good tool for avoiding indeterminacy or explosiveness. One might also wonder, then, whether or not policy inertia has similar effects in the case of optimal policy. The last term in (5) captures the idea of policy inertia in the context of optimal monetary policy. ${ }^{12}$

Finally, we stress that our notion of optimal policy must be understood as ad hoc optimal policy rather than fully optimal policy for two reasons. First, coefficients $\omega_{x}$ and $\omega_{i}$ express the preferences of the central bank. In contrast, under fully optimal policy these two parameters would be a function of the structural parameters of the economy. Second, fully optimal policy would require considering an objective derived from an economy with heterogeneous expectations.

\section{The Conventional Objective}

\subsection{Implementation via Expectations-Based Reaction Function I}

Evans and Honkapohja (2006) illustrate the derivation of an expectationsbased reaction function of optimal policy given $\omega_{i}=0$. One of their main results of the study is that the expectations-based reaction function is the superior implementation strategy under homogeneous private sector expectations, being formed either rational or by adaptive learning. Therefore we focus on the expectationsbased reaction function. ${ }^{13}$

\footnotetext{
${ }^{12}$ See Woodford (2003, p.582ff.) and Giannoni and Woodford (2005, p.106ff.) for details of two theoretical arguments in favour of such an objective. Briefly, these are non-negligible transaction frictions as discussed in Friedman (1969) or punishment of potential violations of the zero lower bound on the policy instrument.

${ }^{13}$ Alternatively the central bank may opt for the so-called fundamentals-based reaction function as its implementation strategy. However, its performance with regard to determinacy and stability under learning is poor even under homogeneous expectations, see e.g. Evans and Honkapohja (2003, 2006, 2009a,b).
} 
Implementation via expectations-based reaction function pivots on the specific targeting rule under commitment from a timeless perspective. This rule is

$$
\pi_{t}=-\frac{\omega_{x}}{\lambda}\left(x_{t}-\alpha x_{t-1}-\beta^{2}(1-\alpha) \theta^{2} E_{t} x_{t+1}\right)
$$

and can be derived via combining the first-order conditions of the central bank's policy problem. ${ }^{14}$

The expectations-based reaction function in Evans and Honkapohja (2006, p.26) explicitly takes into account observed homogeneous private sector expectations, whereas in our case it takes as given the observed heterogeneous private sector expectations. Now, we may combine (6) and (1)-(4), which yields

$$
i_{t}=\delta_{x} E_{t} x_{t+1}+\delta_{\pi} E_{t} \pi_{t+1}+\delta_{L x} x_{t-1}+\delta_{L \pi} \pi_{t-1},
$$

where $\delta_{x} \equiv \sigma\left[\alpha-\frac{\omega_{x}}{\omega_{x}+\lambda^{2}}(1-\alpha)(\beta \theta)^{2}\right], \delta_{\pi} \equiv \alpha\left[1+\frac{\lambda \sigma}{\omega_{x}+\lambda^{2}} \beta\right], \delta_{L x} \equiv \sigma\left[(1-\alpha) \theta^{2}-\right.$ $\left.\frac{\omega_{x}}{\omega_{x}+\lambda^{2}} \alpha\right]$, and $\delta_{L \pi} \equiv\left[1+\frac{\lambda \sigma}{\omega_{x}+\lambda^{2}} \beta\right](1-\alpha) \theta^{2}$. Note that the policy instrument is a function of private sector expectations, as is typical for this implementation strategy. Inspection of (7) also makes clear that, as long as all agents form RE $(\alpha=1)$, the coefficients imply a positive response to RE about the output gap, a more than one-for-one response to $\mathrm{RE}$ about inflation, a negative response to lagged output gap and no response to lagged inflation. However, these coefficients imply an alteration in feedback in case of expectational heterogeneity, $\alpha \in(0,1)$. The latter introduces lagged output gap and inflation into the economy (1)-(2) and coefficients respond to this by: a smaller response to RE about the output gap; a smaller feedback to RE about inflation, eventually less than one-for-one; a

\footnotetext{
${ }^{14}$ We outline the central bank's policy problem in the online appendix which is available on the author's website: www.urleiwand.com.
} 
larger feedback to lagged output gap and a positive feedback to lagged inflation. Be aware that policy potentially violates the form of the Taylor (1993)-principle as discussed in Woodford (2001, p.233), Woodford (2003, p.254), Bullard and Mitra (2002, p.1116), Bullard and Mitra (2007, p.1183), and Evans and Honkapohja (2009a, p.42), since $\left(\delta_{x}+\delta_{L x}\right)(1-\beta) / \lambda+\left(\delta_{\pi}+\delta_{L \pi}\right)<1$ is possible for $\theta<1$.

\subsection{The Numerical Approach to the Analysis}

We base our analysis of reaction functions such as (7) on numerical methods and illustrate so-called regions of local determinacy, indeterminacy, and explosiveness similar to Bullard and Mitra (2002). The different regions are usually plotted in a plane where the axes measure either $\left(\omega_{x}, \theta\right)$ or the monetary policy parameters $\left(\omega_{x}, \omega_{i}\right)$ for different $\theta .{ }^{15}$ We do so because we are dealing with high dimensional economic systems that do not provide analytical conditions for determinacy.

Given a reduced form model (1)-(2) and an implementation strategy, we will usually end up with a second-order difference system of the form

$$
\mathbf{y}_{t}=\mathbf{A} E_{t} \mathbf{y}_{t+1}+\mathbf{C} \mathbf{y}_{t-1}
$$

where $\mathbf{y}_{t}$ is a $m \times 1$ vector of endogenous variables and matrices $\mathbf{A}$ and $\mathbf{C}$ are $m \times m$ matrices. In order to assess the dynamic properties of such a system, one chooses a solution procedure that, as a by-product, yields the eigenvalues of the system matrix. It is precisely these eigenvalues that characterize the system dynamics. One may either apply the solution method of Blanchard and Kahn (1980) or the more robust purely numerical method proposed by Klein (2000).

\footnotetext{
${ }^{15}$ For all cases we provide animated plots for the share of agents with $\mathrm{RE}, \alpha \in[0,1]$ on the author's website: www.urleiwand.com.
} 
The advantage of the latter is that it is based on generalized eigenvalues (GEVs) and allows matrices $\mathbf{A}$ and $\mathbf{C}$ to be singular (see McCallum 2009a).

We can thus calculate GEVs for any combination of the monetary policy parameters. Moreover, we can count the number of GEVs, whose moduli are inside or outside the unit circle for any parameter combination. In particular, at any point within the parameter space, where the number of GEVs whose moduli lie outside the unit circle matches the number of free variables, there is local determinacy. Next, when the number of GEVs whose moduli lie outside the unit circle is less than the number of free variables we have local indeterminacy. ${ }^{16}$ Finally, when the number of GEVs whose moduli lie outside the unit circle exceeds the number of free variables there is local explosiveness. ${ }^{17}$

\subsection{The Calibration of the Economy}

In order to carry out our numerical analysis, we calibrate our model according to Table 1 below. Our choices of $\beta, \lambda$, and $\sigma$, often referred to as the Woodford (1999)-calibration, allow comparability of our results with the ones of Evans and Honkapohja (2006) or Duffy and Xiao (2007) with regard to determinacy.

Furthermore, recall that our analysis considers expectational heterogeneity. In particular, next to the case of only rational agents, $\alpha=1$, we first and foremost study the coexistence of rational and non-rational agents, $\alpha<1$, which in turn puts the parameter $\theta$ into action. This parameter characterizes the type of non-rational expectations. Compared to Branch and McGough (2009, p.1046ff.)

\footnotetext{
${ }^{16}$ Some authors distinguish indeterminacy of different order, in which the order measures the difference between the number of free variables and the number of GEVs whose moduli lie outside the unit circle. For example, when the difference is two, one labels that Order 2 Indeterminacy. This denotes a situation with a system exhibiting a two-dimensional continuum of stationary equilibria. The idea behind this is to indicate "the number of independent sunspots required to specify the solution", see Evans and McGough (2005, p.1816).

${ }^{17}$ In our analysis we ignore the special case, where one or more moduli of the GEVs may lie on the unit circle.
} 
TABLE 1

Calibrations Used In The Numerical Analyses, Quarterly Frequency

\begin{tabular}{lll}
\hline \hline Parameter & \multicolumn{1}{c}{ Description } & \multicolumn{1}{c}{ Value } \\
\hline$\alpha$ & Share of agents with RE & $\in\{1.00, \ldots, 0.10\}$ \\
$\beta$ & Period discount factor & $0.99^{\mathrm{a}}$ \\
$\lambda$ & Parameter relating to the degree of price stickiness & $0.024^{\mathrm{a}}$ \\
$\sigma$ & Inverse of the inter-temporal elasticity of substitution & $0.157^{\mathrm{a}}$ \\
$\theta$ & Forecasting coefficient of type $\gamma=2$ agents & $\in\{0.10, \ldots, 2.00\}^{\mathrm{b}}$ \\
\hline
\end{tabular}

a Taken from Evans and Honkapohja (2006, p.22).

$\mathrm{b}$ The range includes the values used in Branch and McGough (2009, p.1046ff.).

we consider a larger range for $\alpha$, as the degree of expectational heterogeneity in the actual economy is difficult to measure. Branch (2004), for example, provides evidence for heterogeneous expectations amongst agents in micro data that corresponds roughly to $\alpha=0.6$.

\subsection{Main Results}

What are the dynamics like if the central bank sticks to reaction function (7) and the actual economy evolves according to (1)-(4)? In order to answer this question we collect these equations in a system as (8), which is given by

$$
\left[\begin{array}{l}
x_{t} \\
\pi_{t}
\end{array}\right]=\left[\begin{array}{cc}
\frac{\omega_{x}(1-\alpha)(\beta \theta)^{2}}{\omega_{x}+\lambda^{2}} & -\frac{\lambda \beta \alpha}{\omega_{x}+\lambda^{2}} \\
\frac{\lambda \omega_{x}(1-\alpha)(\beta \theta)^{2}}{\omega_{x}+\lambda^{2}} & \frac{\omega_{x} \beta \alpha}{\omega_{x}+\lambda^{2}}
\end{array}\right]\left[\begin{array}{c}
E_{t} x_{t+1} \\
E_{t} \pi_{t+1}
\end{array}\right]+\left[\begin{array}{cc}
\frac{\omega_{x} \alpha}{\omega_{x}+\lambda^{2}} & -\frac{\lambda \beta(1-\alpha) \theta^{2}}{\omega_{x}+\lambda^{2}} \\
\frac{\omega_{x} \lambda \alpha}{\omega_{x}+\lambda^{2}} & \frac{\omega_{x} \beta(1-\alpha) \theta^{2}}{\omega_{x}+\lambda^{2}}
\end{array}\right]\left[\begin{array}{c}
x_{t-1} \\
\pi_{t-1}
\end{array}\right] .
$$

There are two free variables in the system. Thus, if there are two GEVs outside the unit circle, determinacy follows. Figure 1 presents the numerical results. The RE benchmark is illustrated in Panel 1(a), whereas Panel 1(b) has the results for reaction function (7) given the special case of $\alpha=0.5$.

Re Benchmark. In case of $\alpha=1$, determinacy prevails in the complete 


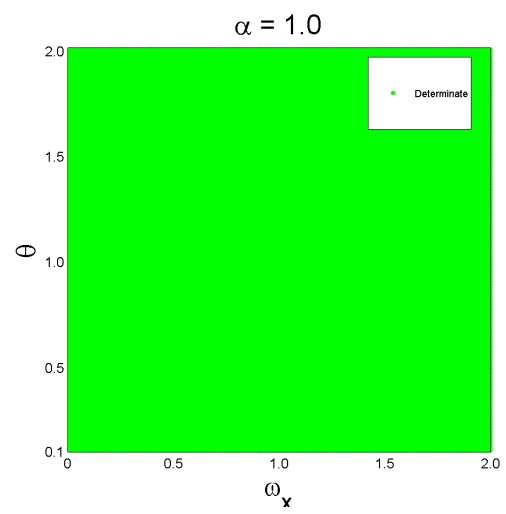

(a) RE benchmark

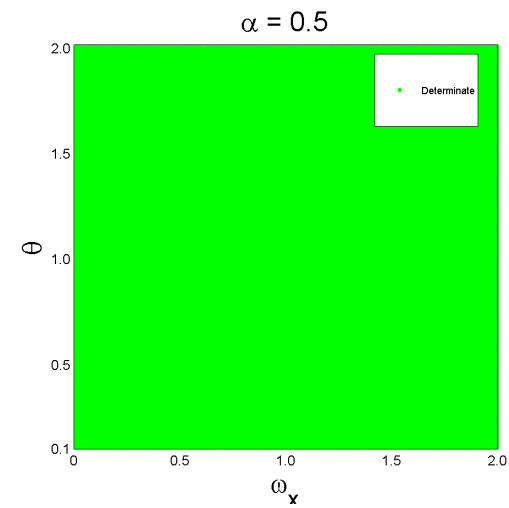

(b) Design based on true model

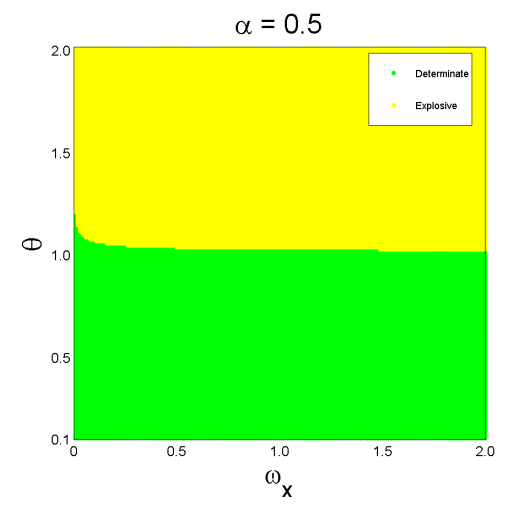

(c) Design based on misspecified model

Fig. 1. Regions of (In-)determinacy and explosiveness for the expectations-based reaction function based on the conventional objective in the $\omega_{x}-\theta$-space for $\alpha \in\{0.5,1.0\}$.

parameter-space considered. This is not a surprise. First, (9) resembles the reduced form in Evans and Honkapohja (2006) in the case of $\alpha=1$. Second, it is well known that optimal monetary policy under commitment in the pure RE version of the NK model yields determinacy, when implemented in this way. For a formal proof see, for example, Evans and Honkapohja (2006, p.35ff.) or Woodford (2003, ch.7).

Heterogeneous Expectations. Our computations indicate that determinacy prevails throughout the considered parameter space. Consequently the 
optimal policy rule under heterogeneous expectations implements the optimal REE and successfully rules out suboptimal stationary REE. This extends the strong result of Evans and Honkapohja (2006) regarding the expectations-based reaction function as the superior implementation strategy to a heterogeneous expectations economy. Likewise this finding suggests a resolution to the threats to economic stability emerging from expectational heterogeneity under simple interest rate rules, as discussed in Branch and McGough (2009) or Massaro (2013).

The intuition for this result is related to the properties of rule (7), as discussed in Subsection 3.1 above. Consider coefficients $\delta_{\pi}$ and $\delta_{L \pi}$, which stipulate the feedback to RE about inflation and lagged inflation respectively. The size of $\delta_{\pi}\left(\delta_{L \pi}\right)$ is negatively (positively) related to $\alpha$. This implies that, as RE about inflation become less pervasive in the economy, the feedback to RE about inflation (lagged inflation) gets smaller (larger). In other words, the policy instrument responds stronger to the type of forecasts that are more relevant to inflation pressures, either directly via (2) or indirectly via the real interest rate in (1). A similar line of reasoning applies with regard to $\delta_{x}$ and $\delta_{L x}$. Note that $x_{t}$ directly affects $\pi_{t}$ in this economy. Again, $i_{t}$ responds stronger to the forecasts of $x_{t}$, which are more relevant to the determination of $x_{t}$ and in turn to $\pi_{t}$.

\section{Robustness to Misspecification in Policy Design}

Herein we assume that the central bank bases optimal policy design on a misspecified RE version of the Phillips curve (2). That is, the central bank aims to implement the rule that would be optimal if all agents had rational expectations. One can verify that the design parallels the one in Section 3. Essentially, this assumption corresponds to $\alpha=1$ in (6). As far as the implementation is concerned, the central bank takes private sector expectations again as given, thus 
we combine (6) for $\alpha=1$, with (1)-(4), which yields

$$
i_{t}=\delta_{x}^{*} E_{t} x_{t+1}+\delta_{\pi} E_{t} \pi_{t+1}+\delta_{L x}^{*} x_{t-1}+\delta_{L \pi} \pi_{t-1},
$$

where $\delta_{x}^{*} \equiv \sigma \alpha, \delta_{\pi} \equiv\left[1+\frac{\lambda \sigma}{\omega_{x}+\lambda^{2}} \beta\right] \alpha, \delta_{L x}^{*} \equiv \sigma\left[(1-\alpha) \theta^{2}-\frac{\omega_{x}}{\omega_{x}+\lambda^{2}}\right]$, and $\delta_{L \pi} \equiv$ $\left[1+\frac{\lambda \sigma}{\omega_{x}+\lambda^{2}} \beta\right](1-\alpha) \theta^{2}$. We observe that the coefficients $\delta_{x}^{*}$ and $\delta_{L x}^{*}$ differ from their counterparts in (7). In particular, for $\alpha<1$ and $\omega_{x}>0$, it follows that $\delta_{x}<\delta_{x}^{*}$ and $\delta_{L x}>\delta_{L x}^{*}{ }^{18}$

\subsection{Comparison to the Main Results}

The resulting reduced form is given by

$$
\left[\begin{array}{l}
x_{t} \\
\pi_{t}
\end{array}\right]=\left[\begin{array}{rr}
0 & -\frac{\lambda \beta \alpha}{\omega_{x}+\lambda^{2}} \\
0 & \frac{\omega_{x} \beta \alpha}{\omega_{x}+\lambda^{2}}
\end{array}\right]\left[\begin{array}{l}
E_{t} x_{t+1} \\
E_{t} \pi_{t+1}
\end{array}\right]+\left[\begin{array}{cc}
\frac{\omega_{x}}{\omega_{x}+\lambda^{2}} & -\frac{\lambda \beta(1-\alpha) \theta^{2}}{\omega_{x}+\lambda^{2}} \\
\frac{\omega_{x} \lambda}{\omega_{x}+\lambda^{2}} & \frac{\omega_{x} \beta(1-\alpha) \theta^{2}}{\omega_{x}+\lambda^{2}}
\end{array}\right]\left[\begin{array}{c}
x_{t-1} \\
\pi_{t-1}
\end{array}\right]
$$

Clearly $x_{t}$ and $\pi_{t}$ no longer depend on RE about output gap. Likewise, the coefficients relating $x_{t}$ and $\pi_{t}$ with $x_{t-1}$ no longer depend on the fraction of agents with RE, $\alpha$. This may have serious consequences for the determinacy properties. The numerical results for (10) are presented in Panels 1(a) and 1(c) of Figure 1.

Re Benchmark. Panel 1(a) indicates that again, as long as all agents form $\mathrm{RE}, \alpha=1$, determinacy prevails in the complete parameter-space considered, as in this case reaction function (7) equals (10).

Heterogeneous Expectations. However in case of expectational heterogeneity, $\alpha<1$, the performance of reaction function (10) can differ.

Purely adaptive or naïve expectations. Given that non-rational forecasts are formed purely adaptively or naïvely, $\theta \leq 1$, we observe a qualitatively similar

\footnotetext{
${ }^{18}$ Given our assumptions on the parameter space, one can verify that the Taylor (1993)principle is always satisfied under $(10)$, since $\left(\delta_{x}^{*}+\delta_{L x}^{*}\right)(1-\beta) / \lambda+\left(\delta_{\pi}+\delta_{L \pi}\right)>1$ for the entire parameter space considered.
} 
pattern. The central bank can still render the economy determinate regardless of its preference within $\omega_{x} \in(0,2]$. Thus, conditional on this particular expectational set-up, our main result even holds under policy design based on a misspecified model. In addition, our finding is also consistent with the result of Evans and Honkapohja (2006), in which the reaction function (10) yields stability under adaptive learning as with decreasing $\alpha$, average expectations become purely adaptive.

Extrapolative expectations. Somewhat worrisome is our finding for the case in which non-rational forecasts are extrapolative, $\theta \in(1.0,2.0]$. It appears that the central bank can trigger local explosiveness for many values of $\omega_{x}$.

Variations of $\alpha$ indicate that, for sufficiently large $\theta>1$, the larger the share of non-rational forecasts, the lower the policy preference $\omega_{x}$ must be for determinacy. Otherwise, optimal policy under RE fails to deliver determinacy. Implicitly this means that the larger the relative weight on inflation stabilization, the greater is the chance to ensure determinacy. This finding is consistent with Orphanides and Williams's (2005) result, that policy should respond more aggressively towards inflation in the presence of heterogeneous information.

However, for $\alpha \in\{0.1, \ldots, 0.5\}$ our results suggest that, contrary to the case of homogeneous RE studied in Evans and Honkapohja (2006), the central bank can no longer render the economy determinate for a certain part of the parameter space. The presence of extrapolative expectations is a threat to determinacy regardless of $\omega_{x}$. The coefficients in reaction function (10) and the resulting reduced form (11) can provide some intuition for this observation. The presence of non-rational agents, $\alpha<1$, induces some differences compared to the case of $\alpha=1$ and, equally important, to our main results reported above.

First, notice that extrapolative expectations, in contrast to purely adaptive 
or naïve expectations, possess the inherent tendency to reinforce cumulative processes away from the REE. Now, as $\alpha$ gets smaller, these tendencies in the economy become stronger. Thus, one may expect a stronger response of the policy instrument to such tendencies in order to contain the economy in the REE. As coefficients $\delta_{\pi}$ and $\delta_{L \pi}$ are as in (7), only the central banks stance regarding the output gap, $\delta_{x}<\delta_{x}^{*}$ and $\delta_{L x}>\delta_{L x}^{*}$, can explain the discrepancy in results.

Second, starting with (1), as $\alpha$ gets smaller, extrapolative expectations regarding the output gap get more relevant for the determination of $x_{t}$. If there is an upward pressure in extrapolative expectations of the output gap, $x_{t}$ tend to increase, and via (2), $\pi_{t}$ does so too. This will feed into expectations of inflation, which in turn tend to lower the real interest rate in (1) and increase $\pi_{t}$ via (2). This, in turn, reinforces the mechanism just described.

Third, optimal monetary policy that renders the economy determinate for given preference $\omega_{x}$, manages to shut down the mechanism just described, by responding sufficiently to the relevant expectations. However, in the current case, despite extrapolative expectations becoming more relevant with smaller $\alpha$, the feedback to these expectations implied by $\delta_{L x}^{*}$ is insufficient and lower compared to the one implied by $\delta_{L x}$. Thus, under (7), the impact of extrapolative expectations on $x_{t}$ is successfully mitigated, whereas this is not the case under (10).

Finally, note that the relative strength of the mechanism discussed above also depends on the coefficient $\lambda$, which is negatively related to the degree of nominal price rigidity and governs the sensitivity of inflation to changes in the output gap. One can show that the smaller is $\lambda$, i.e., the more sticky nominal prices are, the more likely it is that the optimal policy rule under RE fails to deliver determinacy given the policymaker's preference $\omega_{x}>0$ throughout the whole range $\theta \in[0.1,2.0]$, as $\alpha$ decreases. This finding suggests that, in order to 
achieve determinacy in this scenario, the central bank may be required to act as if it would favour a lower weight on output gap stabilization, as it actually does.

Overall, our results for the expectations-based reaction function (10) suggest that in an NK economy with heterogeneous expectations the central bank is unable to control nominal prices throughout the parameter space. This pitfall puts a warning sign on this implementation strategy and contrasts with the Evans and Honkapohja $(2006,2010)$ result for homogeneous expectations and our main results above, obtained for policy design in the correctly specified model.

\subsection{Discussion ${ }^{19}$}

Based on the findings within this section, one may argue that, as long as non-rational expectations are purely adaptive, the policymaker can ignore expectational heterogeneity in the policy design. However, such a behaviour of the central bank raises the issue of time-consistency: the policy rule designed under the misspecified or approximating model will de facto never be satisfied, as the expectational heterogeneity is persistent. ${ }^{20}$ Thus, can the policymaker credibly commit to such a policy rule? Or, wouldn't one expect that the policymaker would deviate and instead change the policy design ${ }^{21}$

Clearly, the policymaker can only credibly commit to the design based on the approximating model, if the actual processes of endogenous variables, resulting

\footnotetext{
${ }^{19}$ I owe particular thanks to an anonymous referee for his thoughtful suggestions that form the basis of this discussion.

${ }^{20}$ This distinguishes the set-up herein from Evans and Honkapohja (2006), where the optimal policy rule designed in the $\mathrm{RE}$ version of the economy is implemented asymptotically as private sector expectations asymptotically converge to RE.

${ }^{21}$ Such concerns are consistent with the literature, which assumes that the policymaker initially possesses a misspecified model, but may uncover the true model over time via leastsquares learning. However, it is routinely assumed that the policymaker's misspecification is unintended and turns out to be transient. Such scenarios are elaborated in Sargent (1999, p.68ff.) for optimal policy and in Carlstrom and Fuerst (2004) for simple interest rate rules. In the former case policy turns out to be time-consistent.
} 
from combining this design with the true model, appear to be equivalent to the ones that he expects to be generated by the designed policy in combination with the approximating model. If not equivalent, it is straightforward to ask, why repeatedly missing the target does not provoke doubts over the policymaker's approximating model? The central bank's doubts about the model could either manifest themselves in terms of parameters of, or variables included in, its perceived law of motion (PLM).

The question of whether doubts about the policymaker's model are justified, can be addressed by asking under which conditions a self-confirming equilibrium, as outlined in Sargent (1999, p.68ff.), Evans and Honkapohja (2001, p.325ff.) and Cho, Williams, and Sargent (2002), exists. ${ }^{22}$ For the purpose of analysis, one has to elaborate the central bank's optimal policy design in the approximating model of the economy, where the doubts are parametrized by some coefficients. The design is implemented into this model which results into the PLM. Next, the policymaker's optimal policy designed under the approximating model, implemented into the true model, yields the so-called actual law of motion (ALM). Accordingly, the policymaker can only credibly commit to its optimal policy, if the ALM is consistent with the PLM.

We have addressed both types of doubts from above. ${ }^{23}$ Our results suggest that committing to an optimal policy rule, designed in an economic model which employs the REH, is time-inconsistent, when the actual economy features persistent expectational heterogeneity. Thus, it cannot be an equilibrium outcome. This finding asserts that in practice it may not be credible for a central bank to

\footnotetext{
${ }^{22}$ According to Evans and Honkapohja (2001) this is an example of a restricted perceptions equilibrium. Although, in the special case of a self-confirming equilibrium the misspecification can only be uncovered out of equilibrium.

${ }^{23}$ The analysis is included in the online appendix.
} 
commit to an optimal policy rule designed within a RE version of a DSGE model.

However, recall that our analysis is conducted in a deterministic set-up. In contrast, in a stochastic version of the model, it may be possible that the approach to optimal policy discussed in this section is time-consistent. Assume that the policymaker utilizes a least-squares learning procedure to estimate the coefficients of the PLM. These coefficients would have to satisfy a least-squares projection of the true model onto the approximating model. If so, then asymptotically, i.e., within a self-confirming equilibrium, the policymaker would not have any reason to have doubts about the approximating model. ${ }^{24}$

\section{The Alternative Objective}

We now study the case of $\omega_{i}>0$. Following Woodford (2003, p.582ff.), we manipulate the first-order conditions of the central bank's problem (elaborated in the online appendix) to yield

$$
\begin{aligned}
i_{t}= & \frac{\gamma_{1}}{\gamma_{0}}\left(x_{t}-x_{t-1}\right)+\frac{\gamma_{2}}{\gamma_{0}} \pi_{t}+\alpha \frac{\gamma_{3}}{\gamma_{0}} i_{t-1}-\alpha^{2} \frac{\gamma_{4}}{\gamma_{0}} i_{t-2} \\
& -\frac{\gamma_{5}}{\gamma_{0}} E_{t} x_{t+1}+\frac{\gamma_{6}}{\gamma_{0}} E_{t} i_{t+1}-\frac{\gamma_{7}}{\gamma_{0}} E_{t} i_{t+2}
\end{aligned}
$$

where $\gamma_{0} \equiv\left(1+2 \alpha(1-\alpha) \theta^{2} \beta\right), \gamma_{1} \equiv \frac{\omega_{x}}{\sigma \omega_{i}}, \gamma_{2} \equiv \frac{\lambda}{\sigma \omega_{i}}, \gamma_{3} \equiv\left(1+\frac{\lambda}{\beta \sigma}+\beta^{-1}\right), \gamma_{4} \equiv$ $\beta^{-1}, \gamma_{5} \equiv \beta^{2}(1-\alpha) \theta^{2} \gamma_{1}, \gamma_{6} \equiv(1-\alpha) \theta^{2}\left(\beta+\frac{\lambda}{\beta \sigma}+\beta^{2}\right)$, and $\gamma_{7} \equiv \beta^{3}(1-\alpha)^{2} \theta^{4}$. For $\alpha=1$, (12) resembles Woodford's (2003) implicit instrument rule. This rule is implicit in the sense that it does not depend on private sector expectations or exogenous shocks. However, for $\alpha<1$ only the latter applies. Furthermore, Woodford (2003) proves that commitment to this rule yields a determinate REE that is optimal from a timeless perspective as long as $\alpha=1$. Moreover, Duffy and

\footnotetext{
${ }^{24}$ An example is the analysis in Sargent (1999, p.68ff.).
} 
Xiao (2007) examine rule (12) for the case in which homogeneous expectations are formed via adaptive learning. They find that it yields stability under learning for a large fraction of the relevant parameter space. This motivates our assessment of rule (12) under heterogeneous expectations, as it is also a possible implementation strategy for the central bank in this scenario.

\subsection{Implementation via Implicit Instrument Rule}

Let us now assume that the central bank commits to (12) in all periods and the economy evolves according to (1) and (2). The resulting system can be expressed as (8). Nevertheless, we report the system in a slightly different way:

$$
\begin{aligned}
{\left[\begin{array}{l}
x_{t} \\
\pi_{t}
\end{array}\right]=\phi_{1}\left[\begin{array}{lr}
\alpha+\frac{\gamma_{5}}{\sigma \gamma_{0}} & \sigma^{-1} \alpha\left(1-\frac{\beta \gamma_{2}}{\gamma_{0}}\right) \\
\lambda\left(\alpha+\frac{\gamma_{5}}{\sigma \gamma_{0}}\right) & \alpha\left[\beta\left(1+\frac{\gamma_{1}}{\sigma \gamma_{0}}\right)+\sigma^{-1} \lambda\right]
\end{array}\right]\left[\begin{array}{c}
E_{t} x_{t+1} \\
E_{t} \pi_{t+1}
\end{array}\right] } \\
+\phi_{1}\left[\begin{array}{rr}
(1-\alpha) \theta^{2}+\frac{\gamma_{1} \alpha}{\sigma \gamma_{0}} & \sigma^{-1}(1-\alpha) \theta^{2}\left(1-\frac{\beta \gamma_{2}}{\gamma_{0}}\right) \\
\lambda\left((1-\alpha) \theta^{2}+\frac{\gamma_{1} \alpha}{\sigma \gamma_{0}}\right) & (1-\alpha) \theta^{2}\left[\beta\left(1+\frac{\gamma_{1}}{\sigma \gamma_{0}}\right)+\sigma^{-1} \lambda\right.
\end{array}\right]\left[\begin{array}{c}
x_{t-1} \\
\pi_{t-1}
\end{array}\right] \\
+\phi_{1}\left[\begin{array}{rr}
\frac{\gamma_{7}}{\sigma \gamma_{0}} & -\frac{\gamma_{6}}{\sigma \gamma_{0}} \\
\frac{\gamma_{7} \lambda}{\sigma \gamma_{0}} & -\frac{\gamma_{6} \lambda}{\sigma \gamma_{0}}
\end{array}\right]\left[\begin{array}{c}
E_{t} i_{t+2} \\
E_{t} i_{t+1}
\end{array}\right]+\phi_{1}\left[\begin{array}{rr}
-\frac{\gamma_{3} \alpha}{\sigma \gamma_{0}} & \frac{\gamma_{4} \alpha^{2}}{\sigma \gamma_{0}} \\
-\lambda \frac{\gamma_{3} \alpha}{\sigma \gamma_{0}} & \lambda \frac{\gamma_{4} \alpha^{2}}{\sigma \gamma_{0}}
\end{array}\right]\left[\begin{array}{c}
i_{t-1} \\
i_{t-2}
\end{array}\right],
\end{aligned}
$$

where $\phi_{1} \equiv\left[1+\sigma^{-1}\left(\frac{\gamma_{1}}{\gamma_{0}}+\frac{\gamma_{2} \lambda}{\sigma \gamma_{0}}\right)\right]^{-1}$.

We focus on the central bank's preferences described by a combination of the two relative weights $\omega_{x} \in(0,2]$ and $\omega_{i} \in(0,2]{ }^{25}$

Re Benchmark. Panel 2(a) indicates that the implicit instrument rule yields determinate outcomes, if the policymaker's preference is approximately below $\omega_{i}=0.4$. In this area of the parameter space, there always exists a pair $\left(\omega_{x}, \omega_{i}\right)$ that delivers determinacy. These findings are in line with Duffy and

\footnotetext{
${ }^{25}$ We provide animated plots in the $\omega_{x}-\omega_{i}$-space for $\alpha \in[0,1]$ and $\theta \in\{0.9,1.0,1.1\}$ for Figures 2 and 3 on the author's website: www.urleiwand.com.
} 


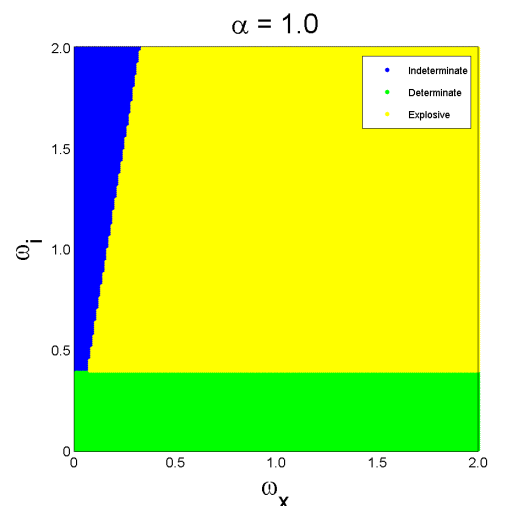

(a) RE benchmark

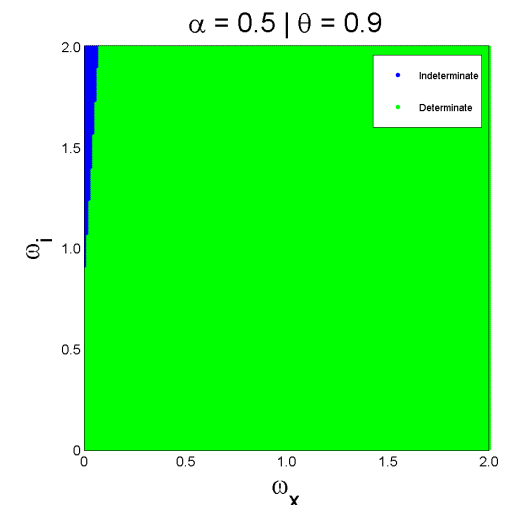

(b) Purely adaptive expectations

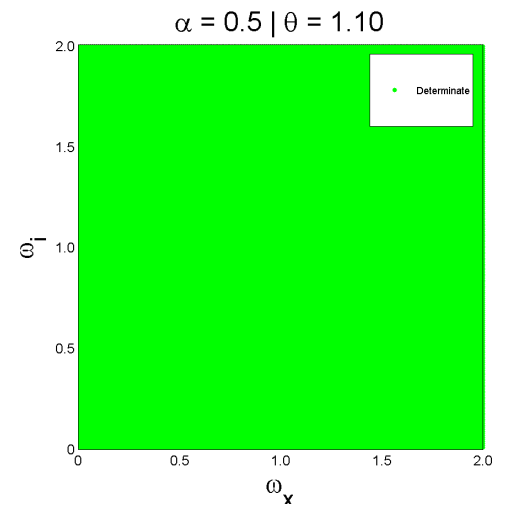

(c) Extrapolative expectations

FiG. 2. Regions of (In-)determinacy and explosiveness for the implicit instrument rule based on the alternative objective in the $\omega_{x}-\omega_{i}$-space for $\alpha \in\{0.5,1.0\}$ and $\theta \in\{0.9,1.1\}$.

Xiao (2007). Moreover, given that explosive paths of the price level or arbitrary large fluctuations are possible, it is obvious that these results are not as strong as the ones obtained by Evans and Honkapohja (2006) for the expectations-based reaction function under the conventional objective that we reproduce above.

Heterogeneous Expectations. Similar to Section 3, our aim now is to gauge the performance under expectational heterogeneity, $\alpha<1$.

Purely adaptive or naïve expectations. Our computations for $\theta=0.9$, as illustrated in Panel 2(b), suggest that the performance of rule (12) improves in 
presence of heterogeneous expectations. For small $\alpha$, any combination of the central bank's preferences $\left(\omega_{x}, \omega_{i}\right)$ effectuates determinacy.

Extrapolative expectations. Panel 2(c) indicates that for the case of extrapolative expectations, $\theta>1$, rule (12) performs better than in the case of purely adaptive or naïve expectations. Moreover, it has similar determinacy properties, as rule (7) related to the conventional objective.

Overall, the implicit instrument rule appears to have an inferior performance compared to that of the expectations-based reaction function above, and questions the merit of policy inertia emerging from the alternative objective. Nevertheless, for the case of $\alpha=1$ it is sometimes argued, that rule (12) is easier to implement, as the central bank does not need to track private sector expectations. However, this operational advantage over an expectations-based reaction function vanishes in case of expectational heterogeneity and on the other hand, the rule requires the central bank to observe contemporaneous values of $\pi_{t}$ and $x_{t}$. Finally, if $\omega_{i} \rightarrow 0$, then both, $\gamma_{1} \rightarrow \infty$ and $\gamma_{2} \rightarrow \infty$. This is discussed in Evans and Honkapohja (2009b) and arguably raises additional concerns about how operational the implicit instrument rule is, and further questions the merit of policy inertia in this context.

\subsection{Implementation via Expectations-Based Reaction Function II}

Inspired by Evans and Honkapohja (2009b, p.158), we consider implementation of the optimal monetary policy based on an expectations-based reaction function comparable to Subsection 3.1. Thus, (12) and (1)-(2) can be combined to yield

$$
\begin{aligned}
& i_{t}= \mu_{x} E_{t} x_{t+1}+\mu_{\pi} E_{t} \pi_{t+1}+\mu_{L 0} x_{t-1}+\mu_{L 3} \pi_{t-1} \\
&+\mu_{i 1} E_{t} i_{t+1}+\mu_{i 2} E_{t} i_{t+2}+\mu_{L 1} i_{t-1}+\mu_{L 2} i_{t-2}, \\
& 24
\end{aligned}
$$


where $\mu_{x} \equiv \frac{\gamma_{0}^{-1}\left[\left(\gamma_{1}+\gamma_{2} \lambda\right)-\gamma_{5}\right]}{\left[1+\left(\sigma \gamma_{0}\right)^{-1}\left(\gamma_{1}+\gamma_{2} \lambda\right)\right]}, \mu_{\pi} \equiv \frac{\gamma_{0}^{-1}\left[\sigma^{-1}\left(\gamma_{1}+\gamma_{2} \lambda\right)+\gamma_{2} \beta\right] \alpha}{\left[1+\left(\sigma \gamma_{0}\right)^{-1}\left(\gamma_{1}+\gamma_{2} \lambda\right)\right]}, \mu_{L 0} \equiv \frac{\gamma_{0}^{-1}\left[\left(\gamma_{1}+\gamma_{2} \lambda\right)(1-\alpha) \theta^{2}-\gamma_{1}\right]}{\left[1+\left(\sigma \gamma_{0}\right)^{-1}\left(\gamma_{1}+\gamma_{2} \lambda\right)\right]}$, $\mu_{L 1} \equiv \frac{\gamma_{0}^{-1} \gamma_{3} \alpha}{\left[1+\left(\sigma \gamma_{0}\right)^{-1}\left(\gamma_{1}+\gamma_{2} \lambda\right)\right]}, \mu_{L 2} \equiv-\frac{\gamma_{0}^{-1} \gamma_{4} \alpha^{2}}{\left[1+\left(\sigma \gamma_{0}\right)^{-1}\left(\gamma_{1}+\gamma_{2} \lambda\right)\right]}, \mu_{L 3} \equiv \frac{\gamma_{0}^{-1}\left[\sigma^{-1}\left(\gamma_{1}+\gamma_{2} \lambda\right)+\gamma_{2} \beta\right](1-\alpha) \theta^{2}}{\left[1+\left(\sigma \gamma_{0}\right)^{-1}\left(\gamma_{1}+\gamma_{2} \lambda\right)\right]}$, $\mu_{i 1} \equiv \frac{\gamma_{0}^{-1} \gamma_{6}}{\left[1+\left(\sigma \gamma_{0}\right)^{-1}\left(\gamma_{1}+\gamma_{2} \lambda\right)\right]}, \mu_{i 2} \equiv-\frac{\gamma_{0}^{-1} \gamma_{7}}{\left[1+\left(\sigma \gamma_{0}\right)^{-1}\left(\gamma_{1}+\gamma_{2} \lambda\right)\right]}$. As in the case of the implicit instrument rule, this alternative expectations-based reaction function now features policy inertia, which could help to stabilize the economy. Reaction function (14) and the actual economy, (1)-(2), can be expressed as a system (8), or as

$$
\begin{aligned}
{\left[\begin{array}{l}
x_{t} \\
\pi_{t}
\end{array}\right]=} & {\left[\begin{array}{lr}
\alpha-\sigma^{-1} \mu_{x} & \sigma^{-1}\left(\alpha-\mu_{\pi}\right) \\
\lambda\left(\alpha-\sigma^{-1} \mu_{x}\right) & \alpha \beta+\lambda \sigma^{-1}\left(\alpha-\mu_{\pi}\right)
\end{array}\right]\left[\begin{array}{l}
E_{t} x_{t+1} \\
E_{t} \pi_{t+1}
\end{array}\right] } \\
+ & {\left[\begin{array}{rr}
(1-\alpha) \theta^{2}-\sigma^{-1} \mu_{L 0} & \sigma^{-1}\left((1-\alpha) \theta^{2}-\mu_{L 3}\right) \\
\lambda\left((1-\alpha) \theta^{2}-\sigma^{-1} \mu_{L 0}\right) & (1-\alpha) \theta^{2} \beta+\lambda \sigma^{-1}\left((1-\alpha) \theta^{2}-\mu_{L 3}\right)
\end{array}\right]\left[\begin{array}{c}
x_{t-1} \\
\pi_{t-1}
\end{array}\right] } \\
+ & {\left[\begin{array}{lr}
-\sigma^{-1} \mu_{i 2} & -\sigma^{-1} \mu_{i 1} \\
-\lambda \sigma^{-1} \mu_{i 2} & -\lambda \sigma^{-1} \mu_{i 1}
\end{array}\right]\left[\begin{array}{c}
E_{t} i_{t+2} \\
E_{t} i_{t+1}
\end{array}\right]+\left[\begin{array}{rr}
-\sigma^{-1} \mu_{L 1} & -\sigma^{-1} \mu_{L 2} \\
-\lambda \sigma^{-1} \mu_{L 1} & -\lambda \sigma^{-1} \mu_{L 2}
\end{array}\right]\left[\begin{array}{c}
i_{t-1} \\
i_{t-2}
\end{array}\right] }
\end{aligned}
$$

Re Benchmark. Results in Panel 3(a) below make it clear that in the case of homogeneous rational agents, there is determinacy throughout the parameter space. This is a new finding, extending the results of Evans and Honkapohja (2009b, p.158) for discretionary policy to the case of policy under commitment. However, the issue of whether this rule yields stability under learning in the homogeneous expectations case remains an open question.

Heterogeneous Expectations. Our focus is on the rule's performance in the case of expectational heterogeneity, $\alpha<1$.

Purely adaptive or naïve expectations. Panel 3(b) indicates that the expectationsbased reaction function will lead to determinacy throughout the parameter space. Although, we consider policy under commitment and heterogeneous expectations, whereas Evans and Honkapohja (2009b, p.158) study the case of discretionary pol- 


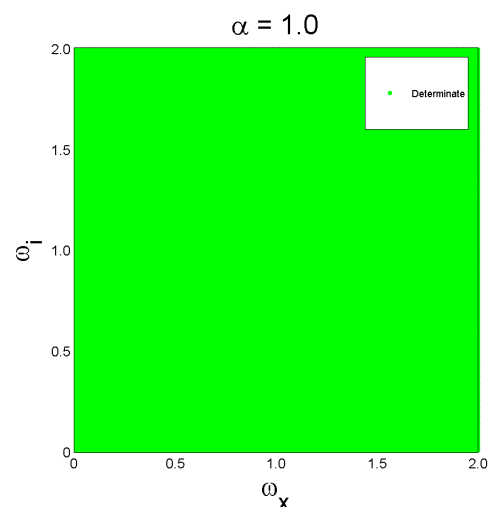

(a) RE benchmark

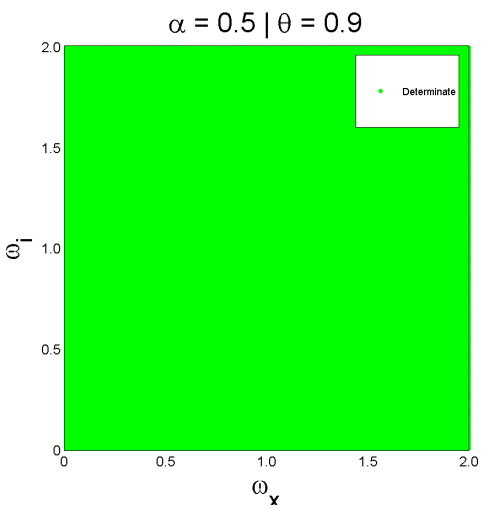

(b) Purely adaptive expectations

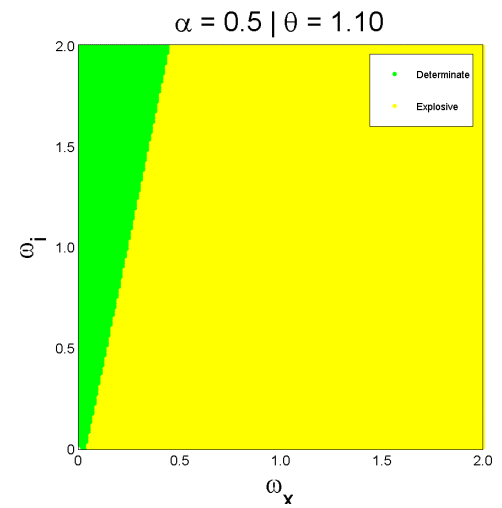

(c) Extrapolative expectations

Fig. 3. Regions of (In-)determinacy and explosiveness for the expectations-based reaction function based on the alternative objective in the $\omega_{x}-\omega_{i}$-space for $\alpha \in\{0.5,1.0\}$ and $\theta \in\{0.9,1.1\}$.

icy under adaptive learning, one can make the case of consistency between these findings. Evans and Honkapohja (2009b, p.158) conclude that the expectationsbased reaction function related to the alternative objective is 'quite robustly stable under learning'. We find that with decreasing $\alpha$, average expectations become purely adaptive and outcomes generated by the same implementation strategy are determinate.

Extrapolative expectations. In contrast, if $\theta>1$, the expectations-based reaction function may lead to either determinacy or local explosiveness depending 
on the central bank's preferences, $\left(\omega_{x}, \omega_{i}\right)$, as well as the share of extrapolators, $(1-\alpha)$. In fact, the determinacy region shrinks with decreasing $\alpha$.

Thus, we conclude that the expectations-based reaction function derived from the alternative objective has an inferior performance compared to the one related to the conventional objective. In the presence of heterogeneous expectations with non-rational extrapolators, there is no clear merit of monetary policy inertia.

\section{Conclusion}

We examine optimal monetary policy designed under heterogeneous expectations in a setting in which the central bank implements the optimal stance via an expectations-based reaction function. The bottom line result of this endeavour is good news for policymakers. When the optimal REE is implemented via an expectations-based reaction function, which appropriately takes into account heterogeneous private-sector expectations, local determinacy prevails. Thus, our main result implies that central banks can successfully rule out suboptimal equilibria in an economy with heterogeneous expectations and are able to fully shield the economy against locally explosive paths and arbitrary large fluctuations.

Next we take up an issue of practical relevance. Can a central bank design optimal monetary policy based on a misspecified model, implement it via an expectations-based reaction function, and still rule out suboptimal REE? The answer is: it depends. It works, if non-rational agents form purely adaptive or naïve expectations. However, if these agents form extrapolative expectations, the central bank runs the risk of losing control over inflation and output in the presence of expectational heterogeneity. In any case, we find that such a policy is not time-consistent. This insight furthers the key implication of our main result that in a world with heterogeneous expectations, the central bank should incorporate 
expectational heterogeneity into the design of optimal monetary policy.

Subsequently we analyze optimal monetary policy when the central bank's objective enforces policy inertia. We examine two implementation strategies, the implicit instrument rule and an alternative expectations-based reaction function. We find that both implementation strategies render the economy determinate for some areas of the parameter space considered, but heterogeneous expectations can pose a serious threat to determinacy. Once the share of non-rational agents is in an empirically plausible range, these implementation strategies may perform poorly compared to ones that generate our main result.

We conclude that the considered implementation strategies fail to completely safeguard the economy against local indeterminacy and explosiveness in the presence of heterogeneous expectations. The merit of policy inertia is far from evident and clear-cut policy recommendations are elusive.

The latter two instability results, for the case when non-rational expectations are purely adaptive or naïve on the one hand, or extrapolative on the other hand, suggest caution regarding popular implementation strategies. Especially as both types of non-rational expectations are empirically documented. See for example Branch (2004) for adaptive beliefs. Moreover, Fuster, Laibson, and Mendel (2010) list evidence for extrapolative beliefs, although they are not as extreme as assumed herein. Put differently, these findings call for a new approach to designing optimal policy when interest-rate stabilization is involved, that can better deal with the potential hazards of expectational heterogeneity. In this setting the question of the merit of policy inertia could then be restated and reexamined. 


\section{References}

Assenza, Tiziana, Peter Heemeijer, Cars H. Hommes, and Domenico Massaro. (2013) "Individual Expectations and Aggregate Macro Behavior." Tinbergen Institute Discussion Paper 16, Tinbergen Institute.

Blanchard, Olivier J., and Charles M. Kahn. (1980) "The Solution of Linear Difference Models under Rational Expectations." Econometrica, 48, 1305-1311.

Branch, William A. (2004) "The Theory of Rationally Heterogeneous Expectations: Evidence from Survey Data on Inflation Expectations." Economic Journal, 114, 592-621.

Branch, William A., and George W. Evans. (2011) "Monetary Policy and Heterogeneous Expectations." Economic Theory, 47, 365-393.

Branch, William A., and Bruce McGough. (2009) "A New Keynesian Model with Heterogeneous Expectations." Journal of Economic Dynamics and Control, 33, 1036-1051.

Bullard, James B., and Kaushik Mitra. (2002) "Learning about Monetary Policy Rules." Journal of Monetary Economics, 49, 1105-1129.

Bullard, James B., and Kaushik Mitra. (2007) "Determinacy, Learnability, and Monetary Policy Inertia." Journal of Money, Credit and Banking, 39, 1177-1212.

Carlstrom, Charles T., and Timothy S. Fuerst. (2004) "Learning and the Central Bank." Journal of Monetary Economics, 51, 327-338.

Cho, In-Koo, Noah Williams, and Thomas J. Sargent. (2002) "Escaping Nash Inflation." Review of Economic Studies, 69, 1-40.

Christiano, Lawrence J., Mathias Trabandt, and Karl Walentin. (2010) "DSGE Models for Monetary Policy Analysis." In Handbook of Monetary Economics, Vol. 3, edited by Benjamin M. Friedman, and Michael Woodford, pp. 285-367, Amsterdam: North Holland.

Clarida, Richard H., Jordi Galí, and Mark Gertler. (1999) "The Science of Monetary Policy: A New Keynesian Perspective." Journal of Economic Literature, 37, 1661-1707.

Cochrane, John H. (2009) "Can Learnability Save New-Keynesian Models?" Journal of Monetary Economics, 56, 1109-1113.

Cochrane, John H. (2011) "Determinacy and Identification with Taylor Rules." Journal of Political Economy, 119, 565-615.

Duffy, John, and Wei Xiao. (2007) "The Value of Interest Rate Stabilization Policies When Agents Are Learning." Journal of Money, Credit and Banking, 39, 2041-2056. 
Evans, George W., and Seppo Honkapohja. (2001) Learning and Expectations in Macroeconomics. Frontiers of Economic Research, Princeton, NJ: Princeton University Press.

Evans, George W., and Seppo Honkapohja. (2003) "Expectations and the Stability Problem for Optimal Monetary Policies." Review of Economic Studies, 70, 807-824.

Evans, George W., and Seppo Honkapohja. (2006) "Monetary Policy, Expectations and Commitment." Scandinavian Journal of Economics, 108, 15-38.

Evans, George W., and Seppo Honkapohja. (2009a) "Expectations, Learning and Monetary Policy: An Overview of Recent Research." In Monetary Policy Under Uncertainty and Learning, Series on Central Banking, Analysis, and Economic Policies, Vol. XIII, edited by Klaus Schmidt-Hebbel, and Carl E. Walsh, pp. 27-76, Santiago, Chile: Central Bank of Chile.

Evans, George W., and Seppo Honkapohja. (2009b) "Robust Learning Stability with Operational Monetary Policy Rules." In Monetary Policy Under Uncertainty and Learning, Series on Central Banking, Analysis, and Economic Policies, Vol. XIII, edited by Klaus SchmidtHebbel, and Carl E. Walsh, pp. 145-170, Santiago, Chile: Central Bank of Chile.

Evans, George W., and Seppo Honkapohja. (2010) "Corrigendum: Monetary Policy, Expectations and Commitment." Scandinavian Journal of Economics, 112, 640-641.

Evans, George W., and Bruce McGough. (2005) "Monetary Policy, Indeterminacy and Learning." Journal of Economic Dynamics and Control, 29, 1809-1840.

Friedman, Milton. (1969) "The Optimum Quantity of Money." In The Optimum Quantity of Money and Other Essays, pp. 1-50, Chicago: Aldine.

Fuster, Andreas, David Laibson, and Brock Mendel. (2010) "Natural Expectations and Macroeconomic Fluctuations." Journal of Economic Perspectives, 24, 67-84.

Galí, Jordi. (2008) Monetary Policy, Inflation, and the Business Cycle: An Introduction to the New Keynesian Framework. Princeton, NJ: Princeton University Press.

Giannoni, Marc P., and Michael Woodford. (2005) "Optimal Inflation-Targeting Rules." In The Inflation-Targeting Debate, Studies in Business Cycles, Vol. 32, edited by Ben S. Bernanke, and Michael Woodford, pp. 93-162, Chicago and London: The University of Chicago Press.

Klein, Paul. (2000) "Using the Generalized Schur Form to Solve a Multivariate Linear Rational Expectations Model." Journal of Economic Dynamics and Control, 24, 1405-1423.

Kydland, Finn E., and Edward C. Prescott. (1977) "Rules Rather than Discretion: The Inconsistency of Optimal Plans." Journal of Political Economy, 85, 473-491. 
Massaro, Domenico. (2013) "Heterogeneous Expectations in Monetary DSGE Models." Journal of Economic Dynamics and Control, 37, 680-692.

McCallum, Bennett T. (2009a) "Causality, Structure, and the Uniqueness of Rational Expectations Equilibria." The Manchester School, 79, 551-566.

McCallum, Bennett T. (2009b) "Inflation Determination with Taylor Rules: Is New Keynesian Analysis Critically Flawed?" Journal of Monetary Economics, 56, 1101-1108.

McCallum, Bennett T. (2009c) "Rejoinder to Cochrane." Journal of Monetary Economics, 56, $1114-1115$.

Orphanides, Athanasios, and John C. Williams. (2005) "Imperfect Knowledge, Inflation Expectations, and Monetary Policy." In The Inflation-Targeting Debate, Studies in Business Cycles, Vol. 32, edited by Ben S. Bernanke, and Michael Woodford, pp. 201-234, Chicago and London: The University of Chicago Press.

Sargent, Thomas J. (1999) The Conquest of American Inflation. Princeton, NJ: Princeton University Press.

Smets, Frank, and Rafael Wouters. (2003) "An Estimated Dynamic Stochastic General Equilibrium Model of the Euro Area." Journal of the European Economic Association, 1, 1207-1238.

Smets, Frank, and Rafael Wouters. (2007) "Shocks and Frictions in US Business Cycles: A Bayesian DSGE Approach.” American Economic Review, 97, 586-606.

Taylor, John B. (1993) "Discretion versus Policy Rules in Practice." Carnegie-Rochester Conference Series on Public Policy, 39, 195-214.

Woodford, Michael. (1999) "Optimal Monetary Policy Inertia." NBER Working Paper 7261, National Bureau of Economic Research.

Woodford, Michael. (2001) "The Taylor Rule and Optimal Monetary Policy." American Economic Review, 91, 232-237.

Woodford, Michael. (2003) Interest and Prices: Foundations of a Theory of Monetary Policy. Princeton, NJ: Princeton University Press. 